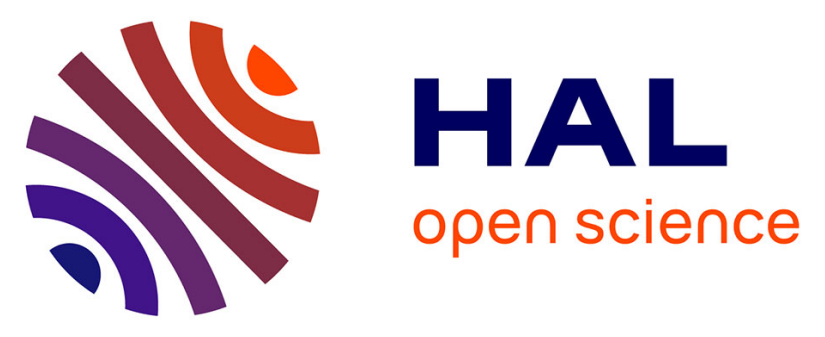

\title{
Serum mesothelin has a higher diagnostic utility than hyaluronic acid in malignant mesothelioma.
}

Bogdan Dragos Grigoriu, Bachar Chahine, Farid Zerimech, Marc Grégoire, Malika Balduyck, Marie-Christine Copin, Patrick Devos, Philippe Lassalle, Arnaud Scherpereel

\section{To cite this version:}

Bogdan Dragos Grigoriu, Bachar Chahine, Farid Zerimech, Marc Grégoire, Malika Balduyck, et al.. Serum mesothelin has a higher diagnostic utility than hyaluronic acid in malignant mesothelioma.. Clinical Biochemistry, 2009, 42 (10-11), pp.1046-50. 10.1016/j.clinbiochem.2009.03.007 . inserm00407520

\section{HAL Id: inserm-00407520 https://www.hal.inserm.fr/inserm-00407520}

Submitted on 25 Jul 2009

HAL is a multi-disciplinary open access archive for the deposit and dissemination of scientific research documents, whether they are published or not. The documents may come from teaching and research institutions in France or abroad, or from public or private research centers.
L'archive ouverte pluridisciplinaire HAL, est destinée au dépôt et à la diffusion de documents scientifiques de niveau recherche, publiés ou non, émanant des établissements d'enseignement et de recherche français ou étrangers, des laboratoires publics ou privés. 
Serum mesothelin has a higher diagnostic utility than Hyaluronic acid in malignant mesothelioma

${ }^{1,8}$ Bogdan Grigoriu, ${ }^{2}$ Bachar Chahine, ${ }^{3}$ Farid Zerimech, ${ }^{4}$ Marc Grégoire, ${ }^{3,5}$ Malika Balduyck, ${ }^{6}$ Marie-Christine Copin, ${ }^{7}$ Patrick Devos, ${ }^{1}$ Philippe Lassalle, ${ }^{1,2}$ Arnaud Scherpereel.

${ }^{1}$ INSERM Unit 774, Institut Pasteur de Lille, Rue du Professeur Calmette, 59000 Lille,

France,

${ }^{2}$ Pulmonary and Thoracic Oncology Department, CHRU of Lille and University of Lille II, ${ }^{3}$ Biochemistry Department, CHRU of Lille, 59000 Lille, France

${ }^{4}$ INSERM U601, 9, quai Moncousu, 44093 Nantes, France

${ }^{5}$ Biochemistry and Molecular Biology Laboratory, Pharmacy School, University of Lille II. 59000 Lille, France

${ }^{6}$ Pathology Department, CHRU of Lille, 59000 Lille, France

${ }^{7}$ Departments of Biostatistics, University of Lille II, 42, rue Paul Duez, 59000 Lille, France

${ }^{8}$ University of Medicine and Pharmacy "Gr.T.Popa" Iasi, Department of Pulmonary

Diseases, Str Universitatii 16, 700115 Iasi, Romania

Corresponding author: Bogdan Dragos GRIGORIU, University of Medicine and Pharmacy

“Gr.T.Popa" Iasi, Department of Pulmonary Diseases, Str Universitatii 16, 700115 Iasi, Romania. Phone: +(40) 7491133 21, Fax:+(40) 2322124 55/+(40) 332810 555, e-mail:

b_grigoriu@hotmail.com

\section{Funding source and conflicts of interest:}

Arnaud Scherpereel is supported by research grants from La Ligue contre le Cancer, Comite de 1'Aisne (2005), Pneumologie Developpement (2004), CIS-Bio internationalß (France) and Fujirebio Diagnostics® (USA).

Bogdan Grigoriu is a recipient of a long term research fellowship from the European Respiratory Society and from the Societe de Pathologie Thoracique du Nord and received a 
travel grant from CIS BIO International and receive funding from the "Consiliul National al Cercetarii Stiintifice in Invatamantul Superior" a governamental research funding agency from Romania.

Bachar Chahine, Farid Zerimech, Marc Grégoire, Malika Balduyck, Marie-Christine Copin, Patrick Devos, and Philippe Lassalle had no conflict of interest to disclose.

CIS-Bio international ${ }^{\circledR}$ and Fujirebio Diagnostics ${ }^{\circledR}$ had no role in recruiting patients, performing assays, analysing the data, and writing or approving the manuscript. 


\begin{abstract}
We assessed comparatively the diagnostic value of two potential malignant pleural mesothelioma (MPM) markers: hyaluronic acid (HA) and soluble mesothelin.

Material and methods: We measured serum and pleural fluid values of mesothelin and hyaluronic acid in 76 patients with MPM, 33 patients with pleural metastases of carcinomas (Mets group) and 27 patients with benign pleural effusion related to asbestos exposure (BPLAE).

Results: Using a serum HA cut-off of $100 \mu \mathrm{g} / \mathrm{L}, 8$ patients/33 $(24.2 \%)$ were positive in the Mets group vs $20 / 76(26.3 \%)$ in the MPM group and only $1 / 27$ BPLAE patients. The area under ROC curve for serum HA in MPM versus Mets or BPLAE groups was only 0.617 while it was 0.755 for mesothelin. In pleural fluid, both markers had similar diagnostic values. Conclusions: Serum mesothelin is more sensitive than hyaluronic acid in diagnosing MPM and there is no benefit in combining both markers.
\end{abstract}

\title{
Keywords
}

Diagnosis, hyaluronic acid, marker, mesothelin mesothelioma, pleural effusion, 


\section{Introduction}

Malignant pleural mesothelioma (MPM) is an aggressive tumor with poor prognosis. Clinical symptoms are not specific and most patients are referred and diagnosed late in the course of the disease. A reliable histologic diagnosis of malignant pleural mesothelioma requires performing multiple pleural biopsies. However many patients are not clinically able to undergo thoracoscopy or transthoracic pleural biopsies. Moreover, the histologic diagnosis may be also difficult due to the broad range of microscopical features of these pleural tumors [1]. Common diagnostic difficulties include differentiation of epithelioid subtype mesothelioma from adenocarcinomas and sarcomatoid subtype mesothelioma from sarcomatoid carcinoma or sarcoma. Therefore, in patients with malignant pleural effusions and a clinical suspicion of MPM, tumor markers would be useful for diagnosis or evaluating prognosis and response to treatment [2]. Hyaluronic acid (HA) has been proposed as a putative diagnostic marker for mesothelioma but high serum levels have been described only in advanced stage mesothelioma [3]. Moreover a significant proportion of mesothelioma do not secrete HA [4, 5]. However high pleural levels of HA (higher than $100 \mathrm{mg} / \mathrm{L}$ ) have been considered to be specific for MPM diagnosis. Despite a quite high area under the ROC curve (0.752) reported by Fuhrman et al. [6], the sensitivity of the test is only about $40 \%$ to $70 \%$ at most [7-10]. More recently soluble mesothelin [11-13] emerged as a potential diagnostic marker for MPM. To our best knowledge, there are no data concerning the concomitant assessment of mesothelin and hyaluronic acid. Historically hyaluronic acid assays relied on High Pressure Liquid Chromatography (HPLC) or on radio-immunologic techniques but an easier ELISA-like technique which uses a specific hyaluronic acid binding protein (HABP) for capture and detection has been described and commercialized [14].

We therefore compared the potential diagnostic utility of hyaluronic acid and soluble mesothelin in a group of patients with pleural effusion and suspected of MPM.

\section{Material and methods}




\section{Patients}

Starting May 2003, we recruited consecutive patients suspected of malignant pleural mesothelioma (MPM) from 20 different pulmonary or thoracic surgery departments from the North and West of France. All these patients had clinical symptoms consistent with this diagnosis, i.e. at least chest pain or dyspnoea associated with pleural thickening and an exsudative pleural effusion. In all patients other causes of pleural effusions were excluded and a definitive pathological diagnosis was established after multiple pleural biopsies, following recent international guidelines [15]. Exclusion criteria were any concomitant infectious disease and previous anti-cancer therapy.

Final diagnosis, based on pleural histology, divided the patients into three groups: 76 patients with confirmed MPM, 27 patients with benign pleural lesions associated with asbestos exposure (BPLAE group), and 33 patients with pleural metastasis of various carcinomas (Mets group). Table I shows the main characteristics of the patients. Some of the patients from the present study were also included in our previous report [13] comparing the diagnostic value of osteopontin and mesothelin.

A standard operating procedure concerning patients sampling and data retrieval was set in place. Serum, as well as pleural fluid samples retrieved without anticoagulant, if available, were collected from each patient and stored at $-80^{\circ} \mathrm{C}$ in aliquots until analyzed. Clinical data and outcome of the patients were also collected after informed consent. The local ethics committee approved the study protocol.

\section{Assaying of tumor markers in serum and pleural effusions}

Hyaluronic acid and soluble mesothelin assays were performed using respectively Hyaluronic Acid (HA) test kit (Corgenix, Inc, Denver, Colorado, USA) and Mesomark (CisBio International, Gif/Yvette, France) kit assays according to the manufacturer instructions.

\section{Statistical analysis}


Results were reported as mean and standard deviation (for age) or as median values with the corresponding interquartile ranges (IQR) (for all other parameters). Comparisons between groups were performed using both Kruskal-Wallis test and a non parametric ANOVA after rank transformation as suggested by Conover [16]. The Bonferroni correction was applied for multiple comparisons in post hoc tests. All tests were two sided and a p value of 0.05 or less was considered significant. Areas under ROC curves (AUC) are reported with their 95\% confidence intervals (CI) in parenthesis. Comparisons of AUC were done as suggested by Hanley using values available for both parameters [17]. Unavailable data was coded as missing.

\section{Results}

Of the 136 patients recruited, $33(24.3 \%)$ had pleural metastasis of various carcinomas (Mets group), $27(19.9 \%)$ had benign pleural lesions associated with asbestos exposure (BPLAE group) and $76(55.8 \%)$ had a malignant pleural mesothelioma (MPM group). The majority (60 cases $-79 \%$ ) had an epithelioid mesothelioma. Sarcomatoid and mixed type tumors were diagnosed in eight cases $(10.5 \%)$ each. Details concerning demographic characteristics are given in table I. The mean age was slightly lower in the BPLAE group but the difference was not statistically significant $(\mathrm{p}=0.09)$. As expected, there was a male gender predominance in the BPLAE $(100 \%)$ and MPM $(80 \%)$ groups due to the occupational nature of the asbestos exposure.

\section{Serum hyaluronic acid and mesothelin levels}

In serum, a significant difference of HA was observed between patients with mesothelioma (median $48.5 \mu \mathrm{g} / \mathrm{L}$ IQR 29.5 - 100.5) and benign cases (median $30.0 \mu \mathrm{g} / \mathrm{L}$ IQR 13.0 - 56.0) $(p=0.009)$ but not with those with pleural metastasis of carcinomas (median $45.0 \mu \mathrm{g} / \mathrm{L}$ IQR 19.0 - 91.0) $(p=0.23)$ (Figure $1 \mathrm{~A})$. The AUC for serum HA in the differential diagnosis of MPM versus all other types of pleural involvement was only of 0.617 (95 CI $0.520-0.714$ ) 
while it was 0.672 (95 CI $0.558-0.785$ ) for the differential diagnosis of malignant (Mets and MPM) and benign pleural lesions (figure $1 \mathrm{C}$ and $1 \mathrm{D}$ respectively).

As already reported, serum mesothelin values were much higher in MPM than in both Mets and BPLAE patients (Figure 1B). Mesothelin had higher AUC than serum HA for differentiating malignant (Mets and MPM) and benign pleural lesions $(0.774,95$ CI $0.681-$ $0.867)$ or MPM versus all other type of pleural involvement $(0.755,95$ CI $0.671-0.840)$ (figure $1 \mathrm{C}$ and 1D).

We tried to assess the optimal cut-off values for HA using the Youden index. The maximum values of the Youden index were 0.24 when differentiating between patients with MPM and other diseases and 0.29 for discriminating malignant and benign pleural involvement. These values corresponded to cut-off values for hyaluronic acid of $26.5 \mu \mathrm{g} / \mathrm{L}$ (sensitivity $=0.83$, specificity $=0.42$ ) and $14.5 \mu \mathrm{g} / \mathrm{L}$ (sensitivity $=0.92$, specificity $=0.37$ ). These very low specificities are of little utility for the diagnostic process. Therefore, we considered a cut-off value of $100 \mu \mathrm{g} / \mathrm{L}$, which resulted in a specificity of 0.85 when differentiating between patients with MPM and those with other diseases and of 0.95 when differentiating between neoplastic and benign pleural involvement respectively.

With this new cut-off, we also wanted to know if serum HA could help the diagnosis of MPM in patients with low serum values of mesothelin (Figure 2). Among the 36 patients with MPM and a serum mesothelin value lower than $1.75 \mathrm{nM}$, a cut-off that give a specificity of at least 0.95 (similar to our previous investigations $[12,13])$, only $6(16.6 \%)$ patients had a serum HA at or above the cutoff of $100 \mu \mathrm{g} / \mathrm{L}$ (with values of 100,101,129,148,153, $330 \mu \mathrm{g} / \mathrm{L}: 4$ epithelioid, 1 mixed type and 1 sarcomatoid MPM). In the Mets group four out of 23 patients $(17.4 \%)$ had a low level of serum mesothelin but a high level of serum HA while only one of the 26 BPLAE patients was in the same situation. Thus serum HA can "rescue" the diagnosis of pleural malignancy in about $15 \%$ of the cases but is unable to differentiate between MPM and Mets groups. 


\section{Hyaluronic acid and mesothelin levels in pleural effusions}

Pleural fluid values for HA and mesothelin were available in only 20 patients in the Mets group, 13 patients in the BPLAE group and 26 patients in the MPM group. Pleural values for pleural HA were grossly 1000 times higher than the corresponding serum values. The median values for pleural HA were higher in patients with MPM (133.2 mg/L IQR 20.5 - 336.2) compared with the other two groups $(11.4 \mathrm{mg} / \mathrm{L} \mathrm{IQR} 8.1-22.8$ for Mets group and $22.1 \mathrm{mg} / \mathrm{L}$ IQR 8.5 - 38.4 for BPLAE Group) (Figure 3A). A similar picture was found for pleural mesothelin with median values of $20.5 \mathrm{nM}$ (IQR 7.45 - 106.8) for MPM patients, $7.3 \mathrm{nM}$ (IQR 2.9 -20.3) for Mets patients and $8.1 \mathrm{nM}$ (IQR 4.0 - 15.3) for BPLAE patients (Figure 3B). The AUC for the two markers when differentiating between MPM and other type of pleural involvement were similar ( 0.832 for mesothelin and 0.810 for HA respectively)(Figure 3C). With a cut-off of $50 \mathrm{nM}$ for mesothelin and $50 \mathrm{mg} / \mathrm{L}$ for HA, a specificity higher than 95 $\%$ can be obtained with a sensitivity between 50 and $60 \%$. When we compared the pleural levels of mesothelin and HA in the same patients (figure 3D) we found that the two variables were highly correlated (Spearman $\mathrm{Rho}=0.647, \mathrm{p}=0.001$ ). There was only one patient with low pleural level of mesothelin which had a high pleural HA value. Therefore, the combination of the two pleural effusion markers in our series seemed of little interest for the diagnosis of MPM.

\section{Discussion}

Malignant pleural mesothelioma is a severe disease with an increasing incidence in both developed and emerging countries. MPM prognosis remains poor despite recent treatments. Survival may be improved by an early diagnosis and a multimodal therapeutic approach. Unfortunately, the clinical picture is not specific and yet there are no validated tools for screening or early diagnosis of MPM. Even in patients with an advanced pleural involvement, the diagnosis can be difficult due to the variability of the histologic aspect of mesothelioma and the potential development of other pleural diseases in asbestos-exposed subjects (benign 
lesions, pleural metastases of adenocarcinoma). Therefore, serum diagnostic markers would be very useful in clinical practice helping to refer the patients suspected of MPM earlier for investigations. Historically hyaluronic acid has been the first proposed marker and recent reports $[11,12]$ suggested that soluble mesothelin can also be useful for this purpose.

In the present investigation, we showed that serum HA is elevated in patients with MPM but this marker had a moderate ability to discriminate between patients with MPM and those with other pleural diseases. The lack of specificity of serum HA is due to the elevated values found in patients with pleural metastases of various carcinomas. Soluble Mesothelin had a higher AUC than HA for MPM diagnosis. However, the most important problem of soluble mesothelin remains the less than optimal sensitivity, which was about $40 \%$ in this study. The best estimates found in the literature are at most $70 \%$ if we aim at a specificity of at least $90 \%$.

The addition of hyaluronic acid to mesothelin assessment in blood could orientate the diagnosis in about $15 \%$ of the cases of MPM which have low mesothelin values. This figure can be regarded as low and the real usefulness in practice is debatable in our opinion, since most of these patients still had to undergo a thoracoscopy. Interestingly this additional value of HA was not restricted to patients with epithelioid subtype of mesothelioma. Blood assessment of HA could be eventually used in patients with a high clinical suspicion of MPM, but without a histological proof of cancer and no prospect of chemotherapy, and a low serum mesothelin value. In a similar way, some authors reported that if diagnosis of mesothelioma is based only on a cytological examination the addition of a pleural HA assay can improve sensitivity without significantly compromising specificity [18]. The assessment of HA in the pleural fluid had a better diagnostic value than in serum. Nevertheless, since HA levels were highly correlated with mesothelin levels in pleural effusion, combining the two markers gave redundant information and therefore would be of limited usefulness in clinical practice. The value of serum HA is further reduced by the fact that the levels of this marker are elevated in 
case of hepatic fibrosis or inflammatory joint diseases such as rheumatoid arthritis. Therefore, we suggest that soluble mesothelin should be the sole marker to be initially investigated. This proposal is also sustained by other authors which reported that combining mesothelin with other potential markers as CYFRA21.1, osteopontin or CA 125 was also not helpful for improving MPM diagnosis [13, 19, 20]

In conclusion, serum mesothelin had a better diagnostic value than serum hyaluronic acid and therefore may be considered as the reference biological marker to which all other candidates should be compared in MPM diagnosis. In pleural effusion, both markers were equally effective in diagnosing MPM. 


\section{References}

1. Ordonez, N.G., What are the current best immunohistochemical markers for the diagnosis of epithelioid mesothelioma? A review and update. Hum Pathol, 2007. 38(1): p. 1-16.

2. Chatterjee, S.K.B.R. Zetter, Cancer biomarkers: knowing the present and predicting the future. Future Oncol, 2005. 1(1): p. 37-50.

3. Frebourg, T., G. Lerebours, B. Delpech, et al., Serum hyaluronate in malignant pleural mesothelioma. Cancer, 1987. 59(12): p. 2104-7.

4. Boersma, A., P. DegandG. Biserte, Hyaluronic acid analysis and the diagnosis of pleural mesothelioma. Bull Eur Physiopathol Respir, 1980. 16(1): p. 41-5.

5. Chiu, B., A. Churg, A. Tengblad, R. PearceW.T. McCaughey, Analysis of hyaluronic acid in the diagnosis of malignant mesothelioma. Cancer, 1984. 54(10): p. 2195-9.

6. Fuhrman, C., J.C. Duche, C. Chouaid, et al., Use of tumor markers for differential diagnosis of mesothelioma and secondary pleural malignancies. Clin Biochem, 2000. 33(5): p. 405-10.

7. Soderblom, T., T. Pettersson, P. Nyberg, et al., High pleural fluid hyaluronan concentrations in rheumatoid arthritis. Eur Respir J, 1999. 13(3): p. 519-22.

8. Atagi, S., M. Ogawara, M. Kawahara, et al., Utility of hyaluronic acid in pleural fluid for differential diagnosis of pleural effusions: likelihood ratios for malignant mesothelioma. Jpn J Clin Oncol, 1997. 27(5): p. 293-7.

9. Martensson, G., A. Thylen, U. LindquistA. Hjerpe, The sensitivity of hyaluronan analysis of pleural fluid from patients with malignant mesothelioma and a comparison of different methods. Cancer, 1994. 73(5): p. 1406-10.

10. Thylen, A., A. HjerpeG. Martensson, Hyaluronan content in pleural fluid as a prognostic factor in patients with malignant pleural mesothelioma. Cancer, 2001. 92(5): p. 1224-30. 
11. Robinson, B.W., J. Creaney, R. Lake, et al., Mesothelin-family proteins and diagnosis of mesothelioma. Lancet, 2003. 362(9396): p. 1612-6.

12. Scherpereel, A., B.D. Grigoriu, M. Conti, et al., Soluble Mesothelin-related Protein in the Diagnosis of Malignant Pleural Mesothelioma. Am. J. Respir. Crit. Care Med., 2006. 173: p. 1155-1160.

13. Grigoriu, B.D., A. Scherpereel, P. Devos, et al., Utility of osteopontin and serum mesothelin in malignant pleural mesothelioma diagnosis and prognosis assessment. Clin Cancer Res, 2007. 13(10): p. 2928-35.

14. Chichibu, K., T. Matsuura, S. ShichijoM.M. Yokoyama, Assay of serum hyaluronic acid in clinical application. Clin Chim Acta, 1989. 181(3): p. 317-23.

15. Galateau-Sallé, F.E., Pathology of Malignant Mesothelioma. 1st edition ed. 2006, New York Springer-Verlag 204.

16. Conover, W.J.R.L. Iman, Analysis of covariance using the rank transformation. Biometrics, 1982. 38(3): p. 715-24.

17. Hanley, J.A.B.J. McNeil, A method of comparing the areas under receiver operating characteristic curves derived from the same cases. Radiology, 1983. 148(3): p. 839-43.

18. Welker, L., M. Muller, O. Holz, et al., Cytological diagnosis of malignant mesothelioma--improvement by additional analysis of hyaluronic acid in pleural effusions. Virchows Arch, 2007. 450(4): p. 455-61.

19. van den Heuvel, M.M., C.M. Korse, J.M.G. BonfrerP. Baas, Non-invasive diagnosis of pleural malignancies: The role of tumour markers. Lung Cancer, 2008. 59(3): p. $350-354$.

20. Creaney, J., I. van Bruggen, M. Hof, et al., Combined CA125 and mesothelin levels for the diagnosis of malignant mesothelioma. Chest, 2007. 132(4): p. 1239-46. 
Table I. Demographic data of the recruited patients

\begin{tabular}{|c|c|c|c|}
\hline & Mets $(n=33)$ & BPLAE $(n=27)$ & MPM $(n=76)$ \\
\hline Age years (mean \pm SD) & $66.7 \pm 10.5$ & $61.4 \pm 10.7$ & $66.1 \pm 9.7$ \\
\hline Male gender $\mathrm{n}(\%)$ & $22(66.7 \%)$ & $27(100 \%)$ & $61(80.3 \%)$ \\
\hline \multicolumn{4}{|l|}{ Diagnosed by } \\
\hline - $\quad$ Blind Pleural Biopsy & - & - & $1(1.3 \%)$ \\
\hline - Thoracoscopy & $24(72.7 \%)$ & $18(66.7 \%)$ & $48(63.1 \%)$ \\
\hline - $\quad$ Surgery (VATS or open surgery) & $6(18.2 \%)$ & $9(33.3 \%)$ & $25(33 \%)$ \\
\hline - Guided biopsy (CT or US) & $3(9.1 \%)$ & - & $2(2.6 \%)$ \\
\hline Confirmed asbestos exposure $n(\%)$ & $11(33.3 \%)$ & $22(81.5 \%)$ & $63(82.9 \%)$ \\
\hline
\end{tabular}

$\mathrm{CT}=$ computed tomography US = Ultrasound; Mets = pleural metastasis of carcinoma; BPLAE $=$ benign pleural lesions associated with asbestos exposure; MPM = malignant pleural mesothelioma, VATS = Video Assisted Thoracic Surgery 
Figure legends

Figure 1 Diagnostic utility of serum hyaluronic acid and mesothelin in patients suspected of malignant pleural mesothelioma (MPM). (A) Serum values of hyaluronic acid and (B) serum mesothelin; the horizontal bar shows the median and the doted horizontal line in pane A the selected cut-off value for Hyaluronic acid (100 $\mu \mathrm{g} / \mathrm{L})$; (C) ROC curve for serum mesothelin and hyaluronic acid for differentiating MPM versus all other pleural effusions and (D) malignant pleural effusions (MPM and ADK) versus benign pleural lesions associated with asbestos exposure (BPLAE) Numbers on the graphs represent cut off values for each marker at the respective inflection points.

Figure 2. Correlations between serum values of mesothelin and hyaluronic acid in patients with MPM (solid squares), pleural metastases of carcinomas (triangles) and benign pleural effusions (circles). The Rho values represent the Spearman correlation; the statistically significance $p$ is two tailed.

Figure 3 Diagnostic value of pleural mesothelin and hyaluronic acid. Pleural levels of (A) hyaluronic acid and (B) mesothelin; horizontal lines represent median values for each group. (C) ROC curves for mesothelin (solid line) and hyaluronic acid (dotted line) for differentiating MPM vs other types of pleural effusions; (D): correlation between pleural values of mesothelin ( $\mathrm{x}$ axis) and hyaluronic acid ( $\mathrm{y}$ axis) in patients with MPM (solid squares), pleural metastases of carcinomas (triangles) and benign pleural effusions (circles). The Rho values represent the Spearman correlation; the statistically significance $p$ is two tailed 